\title{
Application Research of Computer Artificial Intelligence in College Student Sports Autonomous Learning*
}

\author{
Xiaolan $\mathrm{Ge}^{1}$ \\ Air Force Engineering University
}

\author{
Yiwei Yin ${ }^{2}$ \\ Yibin University
}

\author{
Suqiong Feng ${ }^{3}$ \\ Sichuan Agricultural University
}

\begin{abstract}
The shortage of qualified teachers, weak basic knowledge foundation of students, and insufficient awareness of autonomous learning of students are the reasons why the sports level of college students is generally low at this stage. Artificial intelligence (AI) technology, as a discipline to study the laws of human intelligent activities, its application in college physical education combining with Internet can effectively alleviate the problems of insufficient qualified teachers and single teaching mode. This paper first briefly introduces the principle, characteristics and application fields of AI. Then it analyzes the system requirements, overall structure, database requirements and system flow one by one, and uses the BP neural network in the field of AI to carry out the design of student autonomous learning. Finally, the AI-based college student sports autonomous learning system is realized in the Visual Studio integrated open environment, the browser/server system architecture, and the SQL Server database under the Windows platform. The intelligence and autonomy of the learning system provide intelligent, open self-evaluation test and effective learning feedback for college students' learning, which is of great significance to help college students improve their sports level and ease the teaching pressure of teachers.
\end{abstract}

\section{Keywords}

Artificial Intelligence (AI) • College Student Sports • Autonomous Learning • BP Neural Network

\footnotetext{
*This paper 2017 scientific research projects in Education Department of Sichuan Province "Transformation development under the background of local undergraduate colleges and universities teachers construction research - in Sichuan province, for example" of one of the research results, Scientific Research project number: 17sb0615

${ }^{1}$ Air Force Engineering University, Xi'an 710051, China. Email: Lannycomeon@163.com

${ }^{2}$ Correspondence to: Yiwei Yin, Yibin University, Yibin 644000, China. Email: yinyiwei427@sina.com ${ }^{3}$ Suqiong, Feng, Sichuan Agricultural University, Chengdu 611130, China. Email: 2476247436@qq.com Citation: Ge, X. L., Yin, Y. W. (2018). Application Research of Computer Artificial Intelligence in College Student Sports Autonomous Learning. Educational Sciences: Theory \& Practice, 18(5), $2143-2154$. http://dx.doi.org/10.12738/estp.2018.5.114
} 
Ge, Yin / Application Research of Computer Artificial Intelligence in College Student Sports Autonomous Learning

In information age, knowledge dissemination, teaching methods and teaching environment are undergoing unprecedented changes. The traditional teaching methods can no longer meet the requirements of the contemporary society for the cultivation of comprehensive talents, and this demand is especially urgent in the field of physical education (Jia, \& Zhang, 2011). The reform of physical education has been promoted for many years in China, and the traditional test-oriented education mode has not produced much effect on sports. At present, college students' physical education has little effect on the overall sports growth of students. Many college students' sports level stays in the high school sports level, and some even appear to decline. The traditional college student physical education mode needs to be reformed. It is necessary to apply computers, AI, and other technologies to physical education teaching practice to improve students' interest in learning and ease the teaching pressure of teachers (Tilford et al., 2013).

AI technology is a subject that makes computers do the intelligent works that can only be done by humans before (Ventos \& Teytaud, 2017; De Brito, Thévin, Garbay, Boissier, \& Hübner, 2016). As one of the most developed information technologies in the world (Crawford et al., 2015), AI technology has made breakthroughs in the open practices of several aspects such as speech recognition, automation control, enterprise management, and teaching systems. Based on this background, this paper explores the application of AI in college students' sports autonomous learning (Yao \& Liu, 2002). Then it introduces the implicit knowledge library of artificial neural network (ANN) under AI, summarizes the flow of neural network model and neural network algorithm, and establishes a mathematical model of knowledge forgetting by using memory forgetting curve (Broda \& Frank, 2015). Moreover, this paper combines with Visual studio computer programming technology, SQL server database to develop the programming design of sports autonomous learning system based on a B/S system architecture. Through detailed analysis of system requirements, overall structure, database requirements, and system flow, the function module settings of the system and the composition of each part of the system are clarified (Cao, Yang \& Yang, 2013). Finally, the interface of the AI-based sports autonomous learning system is displayed. The system provides students with functions such as self-diagnosis, online learning and practice history. It has a positive effect on improving the sports level of college students, and at the same time opens up a new direction in the field of domestic physical education for AI technology.

\section{Overview of AI and ANN model}

\section{Overview of AI}

$\mathrm{AI}$ is an emerging discipline whose central goal is to make computer performance more comprehensive and perfect, and to implant human intelligent associative thinking mode into computers to make it intelligent (Khokhar, Zin, Mokhtar, \& Pesaran 2015).

Development of AI. The development of AI can be divided into three stages in terms of implementation technology:

(1) Achieve problem solving. Replace human to complete part of the logical reasoning work. For example, the proof of the machine theorem in the 1950 s, the checkers program, the solution of general problem S, and the MYCI disease diagnosis and treatment system in 1972-1976, etc. 
(2) Research on intelligent systems. Intelligent systems can achieve harmonious and unified development with the environment. With the research progress of the fifth generation of computers, the interpretation of AI mainly turned to the field of intelligent computers (Ferrein \& Meyer, 2012).

(3) Develop intelligent systems that have human's cognition and thinking. With ANN as the representative, it has created a field that is biased towards practical use in artificial speech recognition, automatic control systems, and network education.

Practical application in the field of AI. At present, the research and application fields of AI mainly focus on pattern recognition, intelligent databases, robots, ANN and so on. Its practical application areas are mainly divided into: (1) pattern recognition; (2) natural language understanding; (3) automatic theorem proof; (4) program automation; (5) intelligent database system; (6) robot; (7) expert system; (8) ANN; (9) intelligent teaching system.

This paper mainly integrates the application of AI in ANN and intelligent teaching system.

\section{ANN model}

Neural network-based implicit knowledge library. The knowledge of neural network is image-like knowledge. It is implicit, and the knowledge library stores knowledge about network structure and omniscience. This knowledge provides information for reasoning and judgment through neural network learning patterns.

The expression of knowledge is shown in formula (1):

$$
\operatorname{IF}\left(x_{1}, x_{2}, \ldots . x_{n}\right)^{T}=(1,0, \ldots, 0)^{T} \operatorname{THEN}\left(y_{1}, y_{2}, \ldots y_{m}\right)^{T}=(0,1, \ldots, 0)^{T}
$$

Where, $\left(\mathrm{x}_{1}, \mathrm{x}_{2}, \ldots . \mathrm{x}_{\mathrm{n}}\right)^{\mathrm{T}}$ represents the description of the knowledge points after the conversion of some questions in the question bank, $\left(\mathrm{y}_{1}, \mathrm{y}_{2}, \ldots \mathrm{y}_{\mathrm{m}}\right)^{\mathrm{T}}$ represents the diagnose result mode of the knowledge points (Huang, MJ et al., 2006).

According to the system knowledge provided by the sports field experts, the sports knowledge is divided into 15 knowledge points, and a sample test question bank is created for the 15 knowledge points. After ANN completes the model training, the students can complete self-test in the question bank.

Training of neural network. Determine the network structure: The autonomous learning system adopts a three-layer network structure, which are input layer, hidden layer, and output layer. The number of output layers $\mathrm{n}=4,4$ is the binary code representing the state of $0-15$, respectively representing the 15 knowledge points, for each knowledge point, 10 questions are selected as samples, each question has two states, binary code 0 indicates correct, 1 indicates error.

Determine the size of training samples: Under normal circumstances, more samples make the training results easier to be found. But because the size of samples is often limited by objective conditions, the accuracy of the training will also be affected by the size of the samples. According to the rule of thumb, the sample size is generally 5-10 times the total number of network connection weights.

Collection and training of sample set: Students of 2016 from a college are selected for PE testing. Collect 
samples of the 150 questions, 2800 samples are obtained and divided into two groups of 1400 samples each, the two groups are the training sample set and the test sample set. The network adopts a traditional BP algorithm, the gradient descent method (Bos \& Weber, 2017). The main steps are shown in Figure 1:

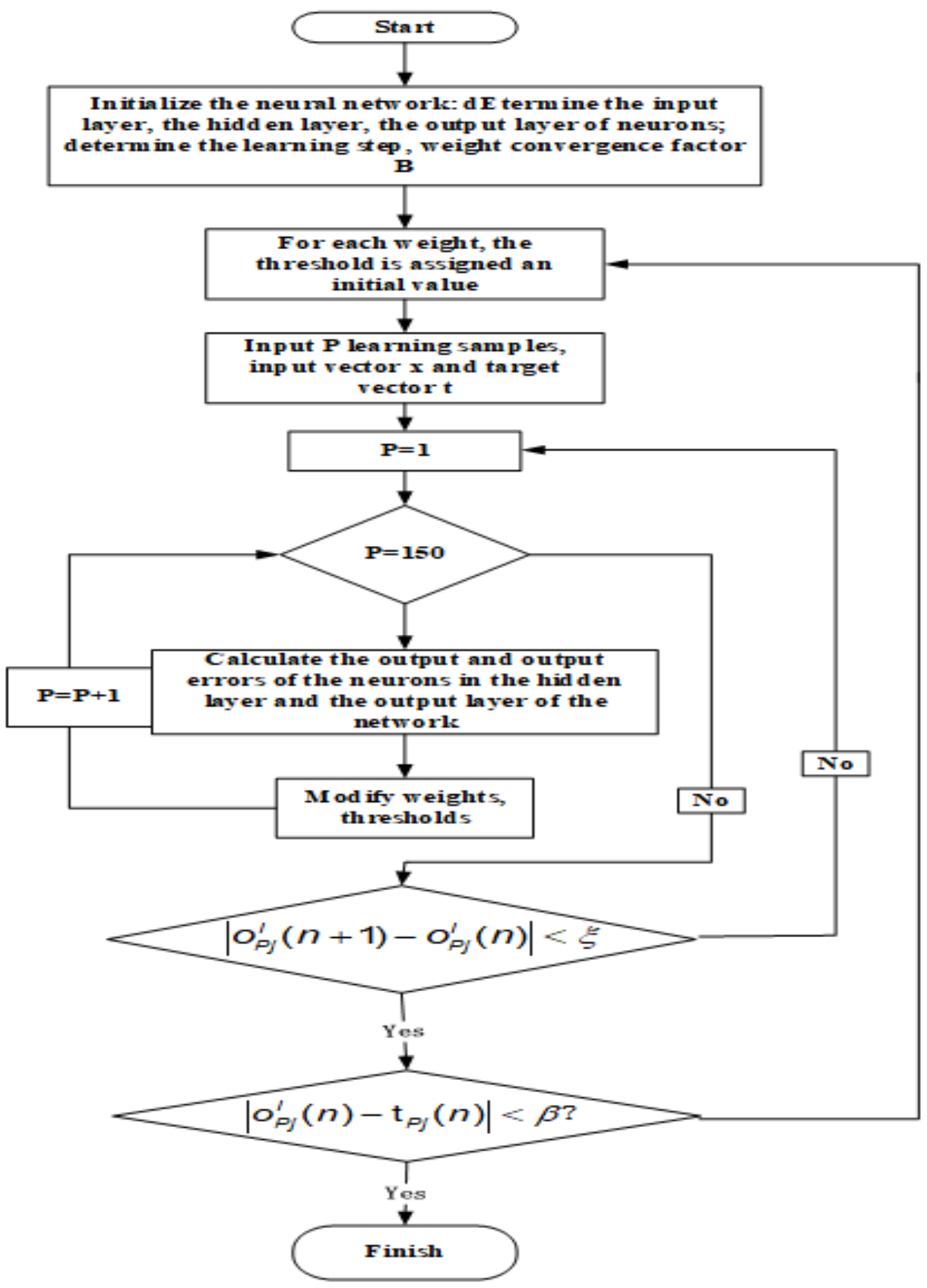

Figure 1. BP training algorithm flow chart 
Knowledge forgetting mathematical modelling. Memory is the process of recognizing, storing, recognizing or recalling past experiences; forgetting is the process by which relevant information cannot be kept or is difficult to be extracted, and can no longer be recognized (Rubaai \& Kankam, 2011). The process of human brain for memorizing and forgetting is shown in Figure 2.

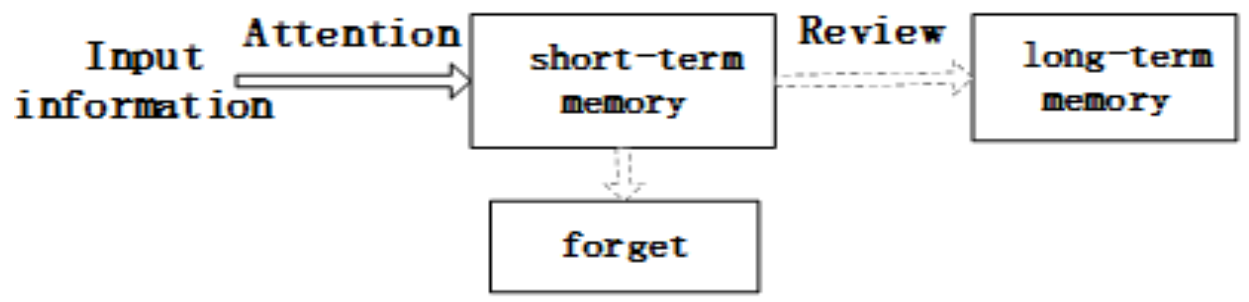

Figure 2. Memorizing and forgetting

In the process of sports learning, the efficiency of information processing should be improved, and shortterm memory should be fully utilized to organize the information of each memory unit so that after each practice the short-term memory would transit to long-term memory. Through the process of attention attraction and repeated connection, the sports knowledge points are mastered by students (Ans \& Rousset, 2000).

Through a lot of research and experiments, scholar Ebbinghaus concluded that the human brain is regular about knowledge forgetting, at the initial stage, the forgetting speed is fast, and then gradually slows down. Therefore, the knowledge of physical education should be reviewed in a timely manner, and it's better to complete the review within one day, otherwise the memory after one day will only be about $30 \%$ of the original. The sports autonomous learning system can provide students with an open-time review platform, which is important for the consolidation of sports knowledge and the promotion of memory (Hu et al, 2013).

\section{System analysis and design}

\section{Overall system design}

The AI-based sports autonomous system emphasizes on its "intelligence", it can make up for the problem of insufficient qualified teachers and give targeted improvement to the weak basic knowledge points of students; it also activates students' autonomous learning enthusiasm, and urges students to arrange learning time effectively and regularly (Weng et al., 2001).

System requirement analysis. There are many difficulties in college physical education classrooms, such as too many students, less class hours, and fewer teachers. It is difficult for students to digest classroom knowledge in time. Due to limited energy, it's also difficult for teachers to give instructions to all students, and they are faced with the heavy tasks of homework correcting. Therefore, the sports autonomous learning system provides a platform for students to timely consolidate and review the sports knowledge, while for teachers, it helps them to reduce the tasks of homework correcting, freeing them to play a more important role in guiding review.

System architecture. The Browser/Server structure is a three-layer structure with a presentation layer, a 
logic layer, and a data layer. It accesses the database through a browser. The three-layer architecture is shown in Figure 3.

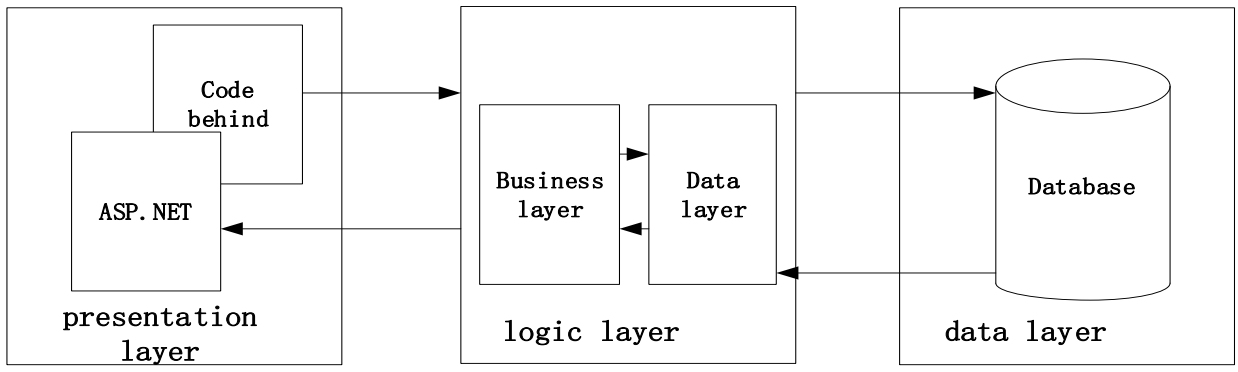

Figure 3. Three-layer architecture system

The three-layer structure has a strict layering method, the data access layer is intelligently accessed by business logic layer, while the business logic layer can only be accessed by the presentation layer, and the user sends the system request through the presentation logic layer, and finally obtain the data through the business logic layer and the data access layer.

\section{System function structure analysis}

There are four types of users in the sports autonomous learning systems: field experts, PE teachers, students, and system administrators.

The main responsibility of the system administrator is to routinely maintain the system, perform query, create and other operations on the user information of students, PE teachers, field expert, etc., and the update and management of question bank is also within the scope of the administrator's work.

The field experts are professors with many years of experience in the sports field. They have a good grasp of sports knowledge points, and can provide samples of the established format for neural network training.

Teachers refer to ordinary college PE teachers, their main responsibility is to organize student examinations, analyze test results and designate the student group's mastery of knowledge points.

Students are subjects who participate in the system for learning. After the registration is authorized, they would participate in practice, self-diagnosis, history practice diagnosis, knowledge points consolidation, selfassessment, and test answer query.

The overall structure of the system is shown in Figure 4. The relationship of various types of users and their main functions are briefly shown in the structure diagram.

\section{System database design}

The system adopts a SQL database, its data storage is mainly completed in the form of data tables. The types of data tables include: user information table, static knowledge table, question bank table, knowledge library table, sample management library table, weight management library table, student history practice library table, and so on. 
The user information table and the student practice table are taken as examples to show the storage mode of the data tables. Table 1 shows the user basic information table, Table 2 shows the student practice table.

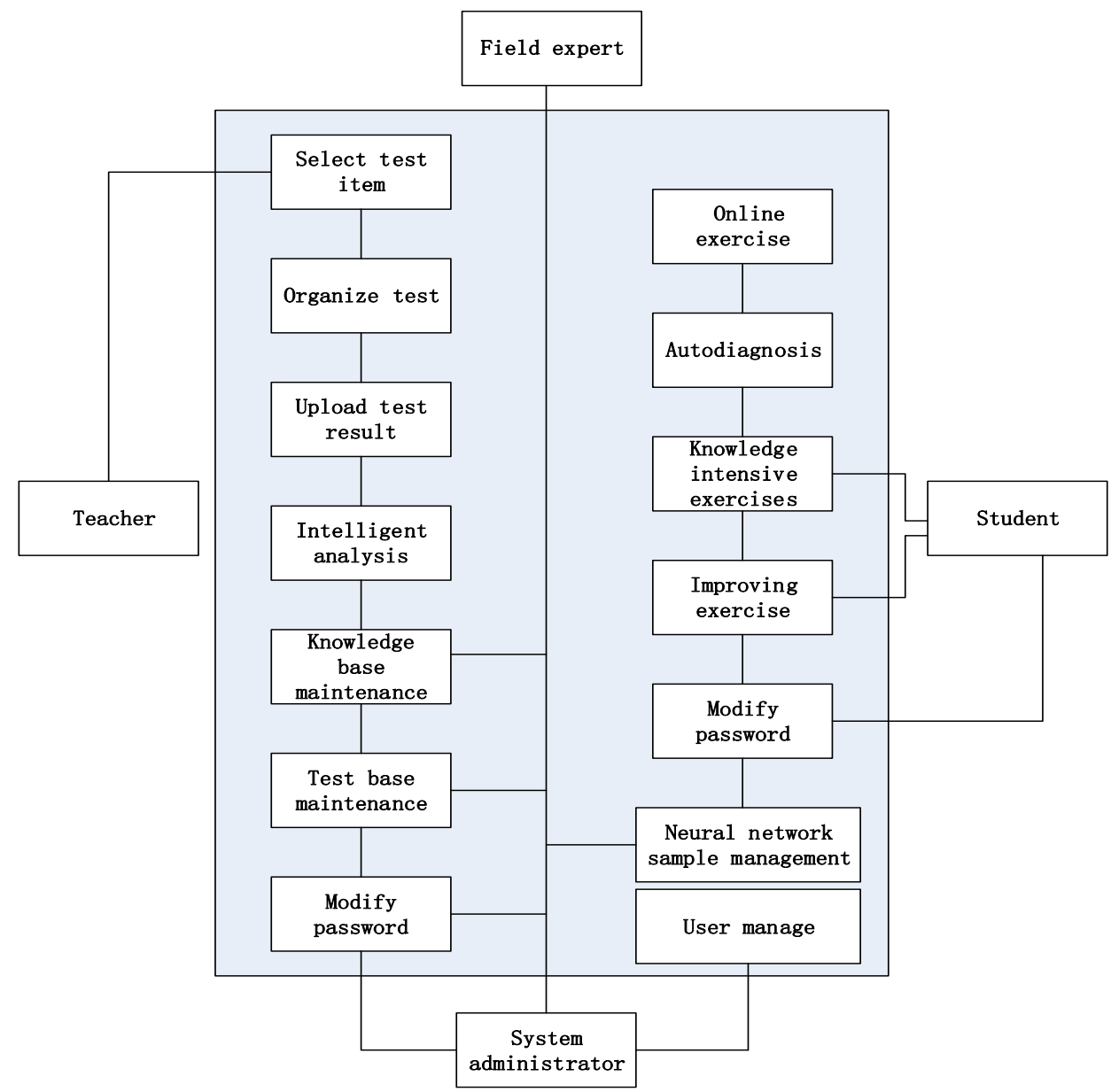

Figure 4. The overall structure of system

Table 1

The User Basic Information Table

\begin{tabular}{lll}
\hline Fieldname & \multicolumn{1}{c}{ Data type } & \multicolumn{1}{c}{ Explanation } \\
\hline Sequence & int & The user's serial number in the system, incremented \\
UserID & varchar & The user name, it is unique in the system \\
Password & varchar & System passw Explanation ord \\
UserType & int & Whether there are lower knowledge points \\
\hline
\end{tabular}

The background configuration of the database design is windows7, the web server uses Internet information server (IIS) 6.0, the development software uses ASP.NET, the background database uses SQL Server 2003, and the database server and the web server are configured to the same computer for development. 
Table 2

The Students Practice Table

\begin{tabular}{lll}
\hline Fieldname & Data type & \multicolumn{1}{c}{ Explanation } \\
\hline StuSequ & int & Student serial number in student table \\
QuestionSequ & int & The original serial number in the gallery \\
Flag & int & Test result right or wrong, 0 that is correct, 1 that error \\
PointSequ & int & Subject to examine the knowledge point serial number \\
Date & datatime & Test time \\
\hline
\end{tabular}

\section{System process flow analysis}

Teachers log in to the system based on their own classroom content, real-time teaching objectives, students' knowledge level, and daily test scores, they can create an exam and organize students to take it. The examination method can be either an offline paper test or an online test in the system. The teachers need to upload the test results through the form of the document, so as to facilitate system's evaluation of students according to schools or classes in later stages.

The process for students to use the autonomous learning system to practice is: first, enter the user name and password and enter the autonomous learning module. For the practices taken by students under the practice mode, the system will give specific evaluation and analysis. During the practice, students can save records and exit, and the next time the system can give students an option to continue the test. After each practice, the system will give a basic evaluation to encourage students to take autonomous practices, and it enhances the fun of learning.

The field experts are mainly responsible for knowledge question creation, specific analysis of questions, options, answers and knowledge points, filling in the confirmation degree, and completing the modification and supplement of the knowledge library.

\section{Realization of college student sports autonomous learning system based on computer artificial intelligence}

Based on the relevant knowledge of ANN based on AI, after giving detailed introduction of system overall design, function requirements analysis, system database analysis and data processing flow, this paper achieves programming and testing of the college student sports autonomous learning system.

Firstly, the system login interface is designed to realize functions such as user system login, password recovery, and new user registration. Figure 4 shows the student login interface of the AI sports autonomous learning system based on the B/S architecture. 


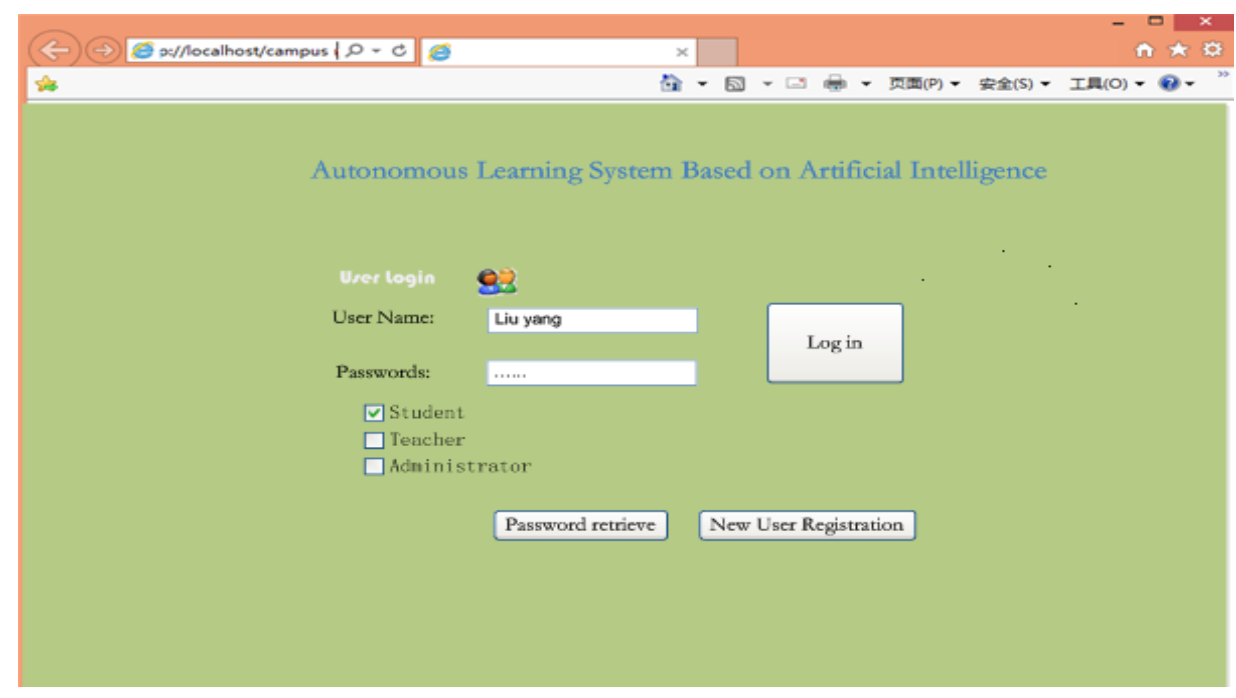

Figure 5. System log-in interface for students

Students can log in to the interface to complete operations of three parts: (1) self-diagnosis; (2) online learning; (3) history practices.

Among them, in online learning, students can choose the corresponding knowledge point module to practice according to their own situation. After completing the exercise, the system will feedback the situation and store it in the database, return the practice analysis results to the students, and provide data for students to complete the analysis of the improved exercises. Figure 2 shows the online test page of the system.

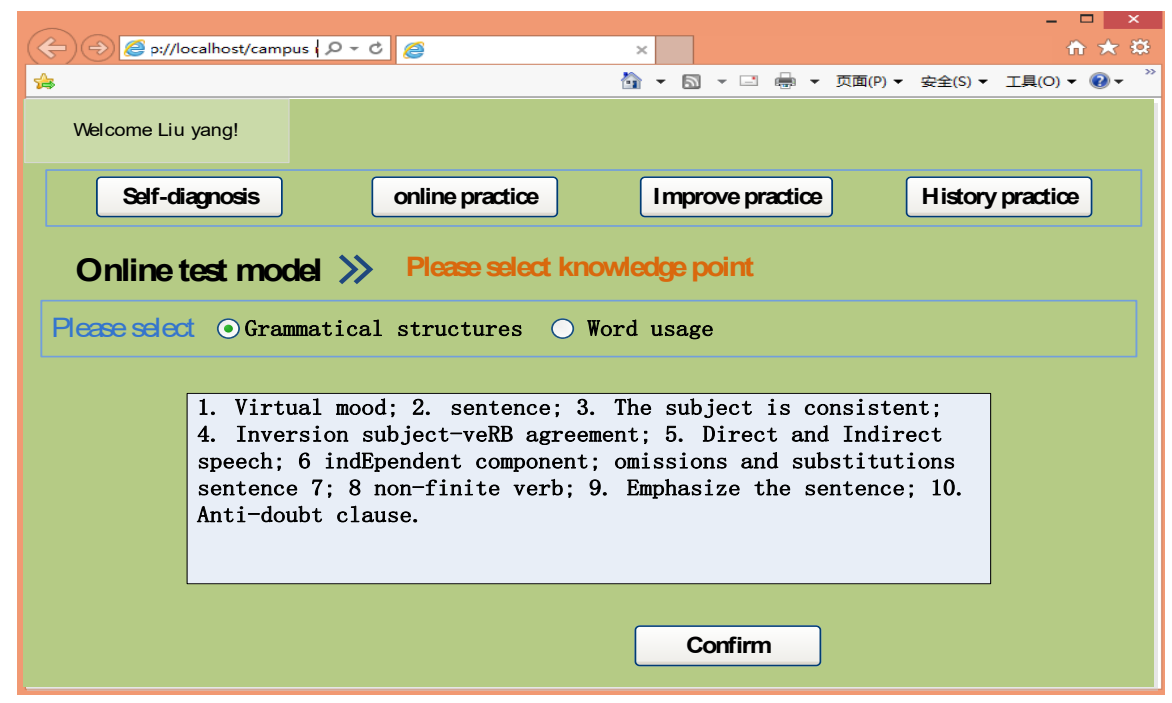

Figure 6. The online test page 
Ge, Yin / Application Research of Computer Artificial Intelligence in College Student Sports Autonomous Learning

In the history practice page, students can view their own practice records, and review the detailed practice situation, improve their learning methods and assist learning according to the learning situation feedback provided by the AI system.

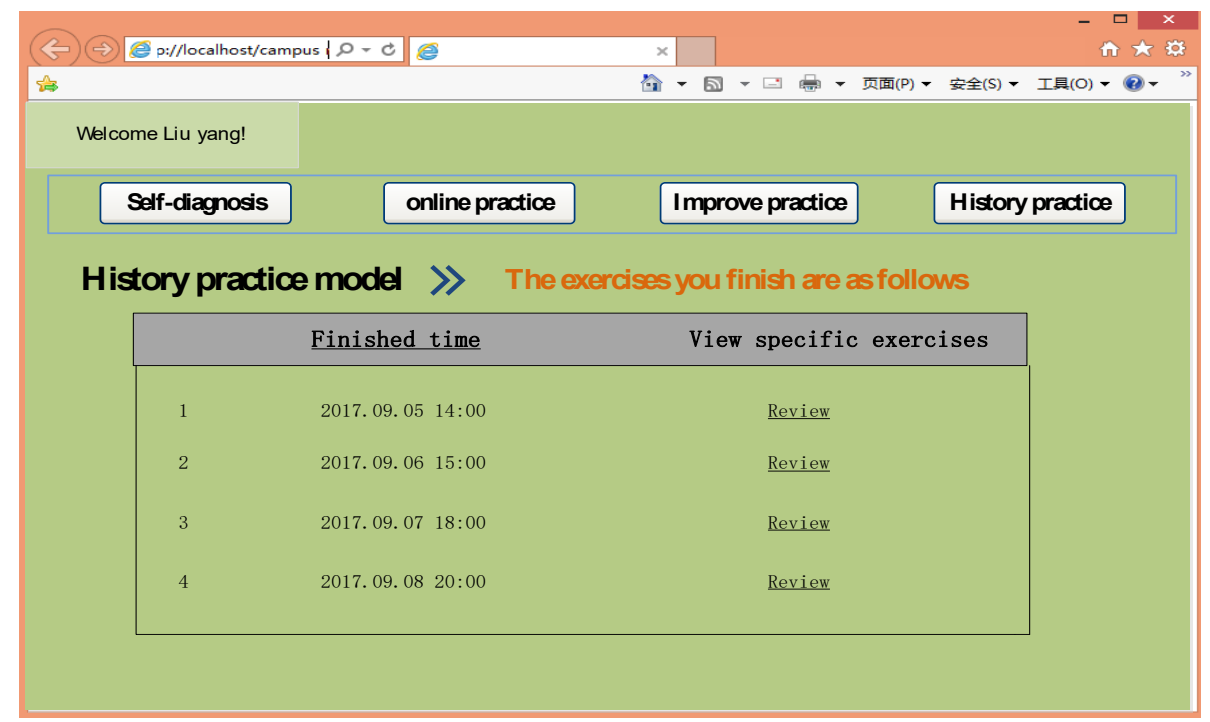

Figure 7. The history practice page

The functions of the teachers and system administrators are also in accordance with the requirements of the system requirement analysis after testing, the pages will not be displayed one by one here. Through the combination of computer technology and AI technology, this paper completes the design and function implementation of the college student sports autonomous learning system.

\section{Conclusion}

This paper introduced the application of computer AI in various fields, and explained the implicit knowledge library and training algorithm of BP neural network. By combining computer programming technology with AI technology, it designed and implemented the AI-based college student sports autonomous learning system. This paper has the following conclusions and application significance:

(1) The design of the system enables teachers to master the basic assessment of the students and achieve control over the overall student sports level of the class, the school and the whole college.

(2) The sports autonomous learning system designed by using ANN can provide independent evaluation for students' practice and test results.

(3) The application of AI in physical education has been promoted, which is of great significance for improving students' autonomous learning in physical education and reducing teachers' teaching pressure. 


\section{References}

Ans, B., \& Rousset, S. (2000). Neural networks with a self-refreshing memory: knowledge transfer in sequential learning tasks without catastrophic forgetting. Connection Science, 12(1), 1-19. https://dx.doi.org/10.1080/095400900116177.

Bos, M., \& Weber, H. T. (2017). Comparison of the training of neural networks for quantitative X-ray fluorescence spectrometry by a genetic algorithm and backward error propagation. Analytica Chimica Acta, 247(1), 97-105. https://dx.doi.org/10.1016/s0003-2670(00)83058-5.

Broda, M., \& Frank, A. (2015). Learning beyond the screen: assessing the impact of reflective artificial intelligence technology on the development of emergent literacy skills. Plant Physiology, 151(2), 681-690.

Cao, Y., Yang, L., \& Yang, Y. (2013). Three-Layer EPR management system architecture and development based on B/S mode. International Symposium on Information Science and Engineering, 8330, 208-211. IEEE. https://dx.doi.org/10.1109/isise.2012.52.

Crawford, E. D., Batuello, J. T., Snow, P., Gamito, E. J., Mcleod, D. G., \& Partin, A. W. (2015). The use of artificial intelligence technology to predict lymph node spread in men with clinically localized prostate carcinoma. $\quad$ Cancer, $\quad 88(9), \quad$ 2105-2109. https://dx.doi.org/10.1002/(sici)10970142(20000501)88:9<2105::aid-cncr16>3.0.co;2-3.

De Brito, M., Thévin, L., Garbay, C., Boissier, O., \& Hübner, J.F. (2016). Situated artificial institution to support the regulation of crisis management, Revue d'Intelligence Artificielle, 30(1-2), 185-209. https://dx.doi.org/10.3166/RIA.30.185-209.

Ferrein, A., \& Meyer, T. (2012). A brief overview of artificial intelligence in south Africa. Ai Magazine, 33(1), 99-101. https://dx.doi.org/10.1609/aimag.v33i1.2357.

Hu, S. G., Liu, Y., Chen, T. P., Liu, Z., Yu, Q., \& Deng, L. J. (2013). Emulating the Ebbinghaus forgetting curve of the human brain with a nio-based memristor. Applied Physics Letters, 103(13), 734. https://doi.org/10.1063/1.4822124

Huang, M. J., Tsou, Y. L., \& Lee, S. C. (2006). Integrating fuzzy data mining and fuzzy artificial neural networks for discovering implicit knowledge. Knowledge-Based Systems, 19(6), 396-403. https://dx.doi.org/10.1016/j.knosys.2006.04.003.

Jia, J., \& Zhang, S. (2011). Study on the management model of foreign language teaching reform of higher education. IEEE, 28(9), 21-29. https://dx.doi.org/10.1109/aimsec.2011.6009850.

Khokhar, S., Zin, A. A. B. M., Mokhtar, A. S. B., \& Pesaran, M. (2015). A comprehensive overview on signal processing and artificial intelligence techniques applications in classification of power quality disturbances. Renewable \& Sustainable Energy Reviews, 51, 1650-1663. https://dx.doi.org/10.1016/j.rser.2015.07.068.

Rubaai, A., \& Kankam, M. D. (2011). Adaptive tracking controller for induction motor drives using online training of neural networks. IEEE Transactions on Industry Applications, 36(5), 12851294.https://dx.dx.doi.org/10.1109/28.871276.

Tilford, S., Clifton, J., Brice, L., Wiggins-Smith, J., Penrose, L., \& Farooqi, N. (2013). Influence of sex education on condom knowledge and use proficiency among Texas college students. Contraception, 88(3), 473-473. https://dx.doi.org/10.1016/j.contraception.2013.05.160. 
Ge, Yin / Application Research of Computer Artificial Intelligence in College Student Sports Autonomous Learning

Ventos, V., \& Teytaud, O. (2017). Bridge: New challenge for artificial intelligence, Revue d'Intelligence Artificielle, 31(3), 249-279. https://dx.doi.org/10.3166/RIA.31.249-279.

Weng, J., Mcclelland, J., Pentland, A., Sporns, O., Stockman, I., \& Sur, M. (2001). Artificial intelligence. autonomous mental development by robots and animals. Science, 291(5504), 599-600. https://dx.doi.org/10.1126/science.291.5504.599.

Yao, X., \& Liu, Y. (2002). A new evolutionary system for evolving artificial neural networks. IEEE Transactions on Neural Networks, 8(3), 694-713. https://dx.doi.org/10.1109/72.572107. 